Proc. Estonian Acad. Sci. Eng., 2005, 11, 2, 126-139

\title{
Particles scattering in particle-wall collisions and its effect on the particle-phase flow
}

\author{
Yury Tsirkunov and Sergei Panfilov \\ Department of Plasma- and Gasdynamics, Baltic State Technical University, 1 1st Krasno- \\ armeiskaya, 190005 St. Petersburg, Russia; tsrknv@bstu.spb.su \\ Received 5 February 2004, in revised form 11 November 2004

\begin{abstract}
The scattering of particles, reflected from a rigid surface, is numerically studied. The effects of surface roughness and particle non-sphericity are analysed separately and in combination. A high-speed subsonic gas-particle flow in a two-dimensional channel with a wedge step is simulated. The particle-phase flow patterns and concentration profiles are obtained for rough wedge surface and for particles, distributed in size. These results are compared with those for smooth wedge surface and monosized particles.
\end{abstract}

Key words: gas-particle flow, particle bouncing, surface roughness, non-spherical particles.

\section{INTRODUCTION}

Modelling two-phase gas-particle flows near solid surfaces must consider some stochastic factors which can be essential in one flow or another. Among these factors are the following:

- roughness of the surface, which randomizes the local particle-wall collision angle and, as a result, the post-collisional particle translational and rotational velocities and the reflection angle;

- non-spherical particle shape, which is also a reason for non-regular reflection of particles;

- size distribution of the particles;

- collisions between particles, which make their motion chaotic;

- turbulence of the carrier gas flow, which randomizes the forces on the particles.

This paper describes in detail the first two factors separately and in combination, and then discusses the interaction between the particles scattering in particlewall collisions and the dispersion of particles in a flow due to their size distribution. 
High-speed flow in a horizontal channel with a wedge step is investigated numerically. For this flow an estimate is obtained for the upper limit of the particle concentration, at which the assumption of a dilute one-way coupled flow is valid. Coarse-grained particles, considered in the present study, move in a channel following "ballistic" trajectories, so that the turbulent fluctuations of the carrier gas flow parameters play no role. In calculations, the wedge surface roughness, the particle size and the mainstream velocity have been taken close to those in experiments $\left[{ }^{1}\right]$.

\section{PARTICLES SCATTERING IN PARTICLE-WALL COLLISIONS}

There are two physical reasons for irregular reflection of particles, colliding with a solid surface: the surface roughness and the non-spherical shape of particles (see, e.g. Chapter 5 in $\left[{ }^{2}\right]$ ). We consider these factors at first separately and then in combination.

\subsection{Effect of the surface roughness}

The roughness is usually a result of the manufacturing process. However, in high-speed flows, in which the velocity of particle-wall collisions exceeds several tens of meters per second (e.g. about $50 \mathrm{~m} / \mathrm{s}$ for hard particles and a ductile metal wall), an essential roughness appears in the process of erosion of a surface by multiple particle impacts. The photo in Fig. 1 demonstrates the erosive effect of a high-speed gas flow, containing corundum particles, on a wedge of ductile metal after several minutes. A schematic view of a wedge in the test section of a closed-jet two-phase wind tunnel is shown in Fig. 2. The wedge angle is equal to $30^{\circ}$, the mainstream particle velocity $V_{\infty}=200 \mathrm{~m} / \mathrm{s}$ and the particle radius $r_{\mathrm{p}}=16 \mu \mathrm{m}$. The clearly visible roughness relief has the form of waves with long
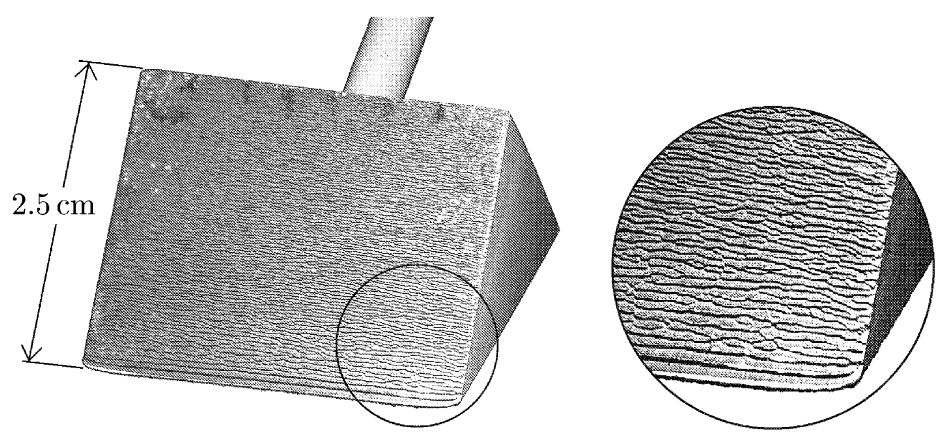

Fig. 1. Wedge of a ductile metal after being subjected to a high-speed gas flow containing corundum particles. 


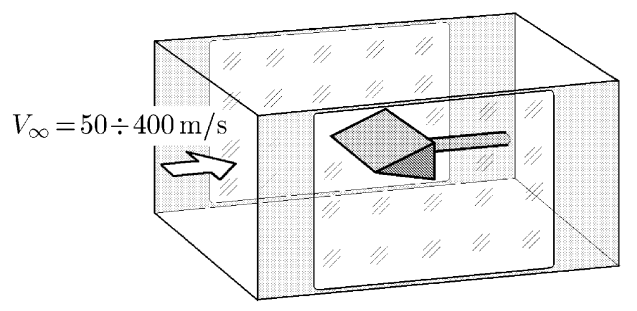

Fig. 2. Schematic view of a wedge in the test section of a wind tunnel.

crests oriented across the flow. Such roughness can be described approximately by a two-dimensional profile in a plane, normal to the leading edge of a wedge. The local collision angle of a particle with a rough surface is obviously random. Therefore the post-collisional particle velocity and the particle reflection angle are also random, the reflected particles are scattered even if they are spheres and their impact velocity and the angle of incidence are fixed. The reflection of spherical particles from a rough wall has been studied in $\left[^{3-6}\right]$. In the two-dimensional collision model, proposed in $\left[{ }^{3}\right]$, the local collision angle was represented as a sum of a regular angle of incidence $\alpha_{1}$ and a random local angle $\gamma$ of surface inclination. The value of $\gamma$ was assumed to satisfy the Gaussian distribution with a mean value of $0^{\circ}$ and a standard deviation $\sigma_{\gamma}$ that should be chosen from the agreement between the numerical and experimental results. Negative values of the collision angle $\alpha_{1}+\gamma$ were considered as physically impossible. This model was extended to the case of a three-dimensional surface roughness in $\left[{ }^{4}\right]$. Subsequent measurements of actual two-dimensional roughness profiles showed that the distribution of $\gamma$ differed from the Gaussian law at $\alpha_{1} \lesssim 15^{\circ}$, and the difference was the greater, the smaller was $\alpha_{1}\left[{ }^{5}\right]$. A collisional model with a distribution of the collisional angle, depending on the angle of incidence $\alpha_{1}$, was proposed in $\left[{ }^{6}\right]$. This dependence was determined from precise measurements of roughness profiles.

Our approach uses a direct numerical simulation of particle-wall collisions. It is based on the detailed geometry of a surface profile, and no assumptions are made for the distribution of a local angle of surface inclination.

Consider a roughness that results from the erosive action of particles. The actual surface profile shown in Fig. 1 was measured with a "Rank Taylor Hobson" profilometer across several longitudinal sections of the wedge. The configuration of the measured profile is presented in Fig. 3a (in this figure the axis $x$ is directed along the wedge surface and $y$ normal to it; note that the scales along the axes are different). We treat a roughness profile as a quasi-periodic function $y(x)$ with randomly varying phase and amplitude and use the following algorithm (proposed earlier in $\left[{ }^{7}\right]$ ) for numerical generation of a stochastic function $y(x)$. In the plane $x, y$ the sequence of $N$ points is set, with coordinates $x_{1}=0, x_{i}=x_{i-1}+\xi$ $(i=2, \ldots, N)$, and $y_{i}=\eta(i=1, \ldots, N)$, where $\xi$ and $\eta$ are Gaussian distributed random variables with means and standard deviations given as $M_{\xi}=h / 2, \sigma_{\xi}<$ $h / 6, M_{\eta}=0, \sigma_{\eta} \leq y_{\max } / 3$. Parameters $h$ and $y_{\max }$ correspond to the mean step 

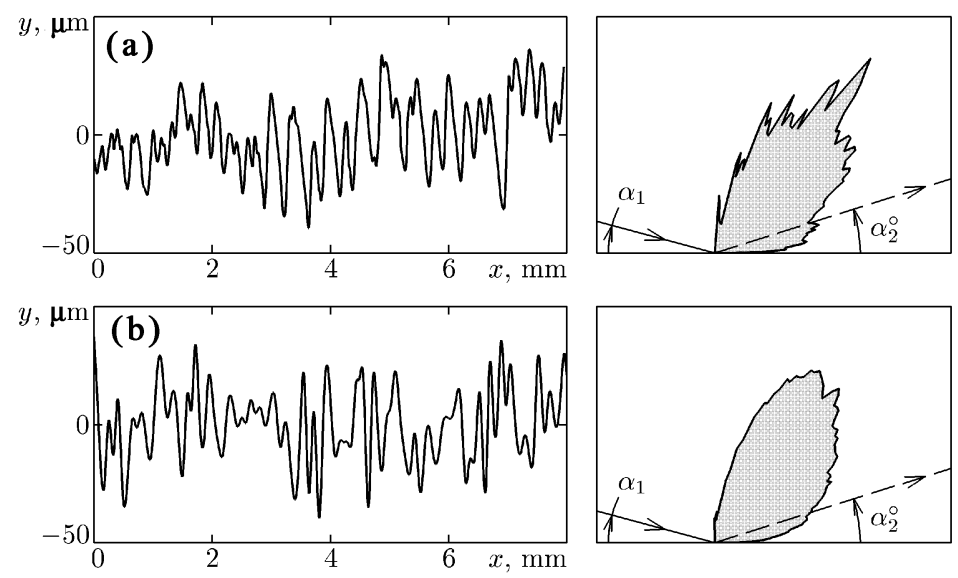

Fig. 3. Configuration of the actual (a) and generated (b) roughness profile (left) and corresponding particle scattering indicatrixes (right); $V_{\mathrm{p} 1}=200 \mathrm{~m} / \mathrm{s}, r_{\mathrm{p}}=16 \mu \mathrm{m}, \alpha_{1}=15^{\circ}$; $\alpha_{2}^{\circ}$ is the angle of particle reflection from a smooth surface.

and maximal height of profile peaks. The values of $x_{i}$ and $y_{i}$, which fall out of the intervals $\left[x_{i-1}+h / 2-3 \sigma_{\xi}, x_{i-1}+h / 2+3 \sigma_{\xi}\right]$ and $\left[-3 \sigma_{\eta},+3 \sigma_{\eta}\right]$, respectively, are ignored, and the sample is repeated. Then we construct a cubic spline through this set of points, which is defined as the roughness profile. A sample of the profile, generated with the statistical values $M_{\xi}=80 \mu \mathrm{m}, \sigma_{\xi}=10 \mu \mathrm{m}$ and $\sigma_{\eta}=20 \mu \mathrm{m}$, is shown in Fig. 3b.

The main objective of roughness profile simulation in modelling of a twophase flow over a rough surface is to describe the scattering properties of the actual surface. One of the most important parameters here is the particle reflection angle $\alpha_{2}$ (the angle between the velocity vector of the reflected particle and the mean line $y=0$ ). The probability density function (PDF) of this angle at the fixed particle impact velocity $V_{\mathrm{p} 1}$ and the angle of incidence $\alpha_{1}$ describe the scattering of reflected particles. The PDF, plotted in polar coordinates $\left(\alpha_{2}\right.$ is the polar angle, PDF is the polar radius), represents a particle scattering indicatrix, which gives an illustrative description of the probability of the particle reflection in different directions.

We use the following technique for the calculation of the PDF. The position of an incident particle is sampled randomly from the uniform distribution and the local collision angle is determined. Then we calculate the post-collisional particle velocity and the local angle of its rebound relative to the local profile inclination, using the semi-empirical model developed in $\left[{ }^{8}\right]$ (this model is valid at a high particle impact velocity). For some parameters, a test particle after its first rebound can collide with the wall again within one hole of the roughness profile. Considering the particle kinematics and the detailed geometry of a profile we calculate the possible second, third etc. collisions before a particle reflects fully 
from a surface and flies away. Note that ignoring such double or multiple collisions may cause a large error in calculation of the reflected angle $\alpha_{2}$ (which can take even a negative value that is physically impossible). Repeating this procedure for a large number of test particles and sequential statistical processing of the results gives the PDF of $\alpha_{2}$. In our calculations, the number of test particles was about $10^{5}$.

The scattering indicatrixes, calculated for spherical particles, and the roughness profiles, shown at the left of Fig. 3, are displayed on the right of Fig. 3. The particle impact velocity $V_{\mathrm{p} 1}$ and the particle radius $r_{\mathrm{p}}$ were taken equal to 200 $\mathrm{m} / \mathrm{s}$ and $16 \mu \mathrm{m}$, respectively. By and large the indicatrixes obtained for the actual and numerically generated profiles are close to each other and hence the scattering properties of these profiles are in close agreement. Numerous peaks on the indicatrix, corresponding to the actual profile, are explained by the small length of the wedge side in experiments, which is not sufficient to be statistically representative. In calculations, it was equal to $2.5 \mathrm{~cm}$ (Fig. 1), whereas the length of the generated profile was equal to $20 \mathrm{~cm}$. As is seen from Fig. 3, the dominant direction of particle reflection from a rough surface differs greatly from that from a smooth surface (the dashed lines).

\subsection{Particle shape effects}

Most actual two-phase gas-particle flows contain non-spherical particles. Such particles are scattered when reflecting from a rigid surface even if the surface is smooth. A general approach to solving the problem of the reflection of an arbitrary shaped particle from a smooth surface is outlined in $\left[{ }^{2}\right]$, Chapter 5 . The problem is three-dimensional and it is very difficult to solve for an arbitrary nonspherical particle having three components of translational and angular velocity. There are very few adequate models of the impact of arbitrary shaped particles on a surface $\left[{ }^{9}\right]$. A collision model becomes more complicated as the impact velocities increase. However, we do not have to solve the general problem in order to obtain useful estimates for scattering. Therefore we use a two-dimensional simplification to investigate the effect of the particle shape on the scattering from both smooth and rough surfaces. We assume that the plane of particle motion coincides with its plane of symmetry. In addition, we simplify the impact model: we assume that the particles do not slide on the surface during collision (i.e., the restitution coefficient of the tangential component of the particle contact point velocity is zero), and the restitution coefficient of the normal component of the particle contact point velocity $a_{n C}$ has a constant value. In calculations it was taken equal to 0.8 that is a typical value for angles of incidence in the vicinity of $\alpha_{1}=15^{\circ}$ in a wide range of the impact velocity.

Using this simplified collision model, we considered four different shapes of particles: sphere, ellipsoid of revolution with a small eccentricity, cube and parallelepiped. Figures 4 and 5 show the scattering indicatrixes of particles, reflected from the smooth and rough surfaces, respectively. 

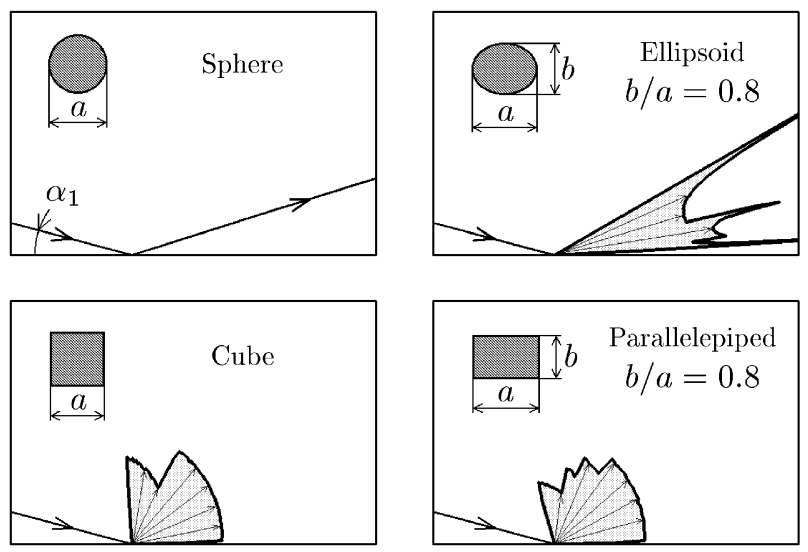

Fig. 4. Scattering indicatrixes for particles of different shape, reflected from a smooth surface; $\alpha_{1}=15^{\circ}, a=32 \mu \mathrm{m}$.
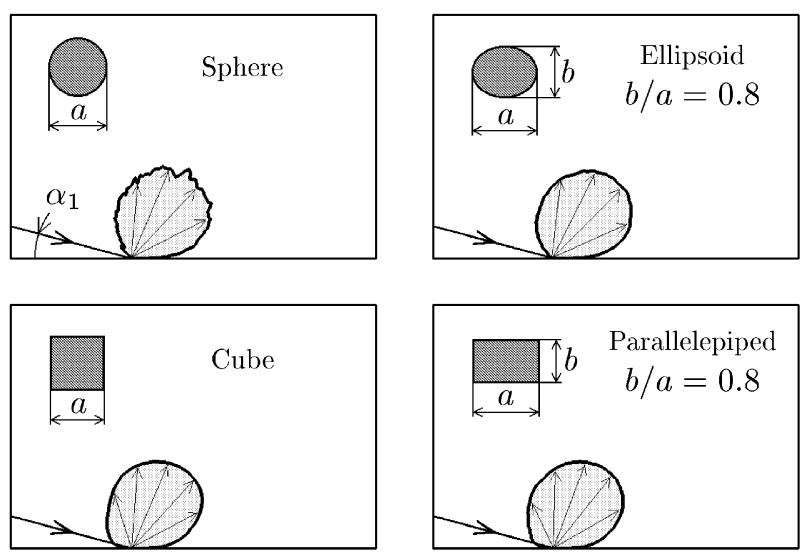

Fig. 5. Scattering indicatrixes for particles of different shape, reflected from a rough surface; $\alpha_{1}=15^{\circ}, a=32 \mu \mathrm{m}$.

The obtained results demonstrate clearly that the shape of particles has a significant effect on how they scatter when reflected from a smooth surface. This effect disappears when the surface is rough, so we can ignore particle shape and, for simplicity, assume them to be spherical when modelling scattering from rough surfaces. Note that we considered small particles as compared with the scale of roughness, and the ratio of major to minor particle dimensions was of the order of unity. The above conclusion is very important for modelling two-phase flows. 


\section{EFFECT OF PARTICLE SCATTERING ON THE PARTICLE-PHASE FLOW}

Besides scattering of reflected particles, another cause of particle mixing in a particle-laden flow is the particle size distribution. Particles of different radii have different inertia and move in a gas flow with a different velocity lag. Even if they impact with the smooth surface of an obstacle at the same point, their angles of incidence and impact velocities are different, and hence, their post-collisional parameters are also different. This results in a redistribution of the particles in a flow that can be also interpreted as particle dispersion. We consider the effect of particle size distribution in combination with particle scattering due to surface roughness on the particle-phase flow structure by an example of a high-speed subsonic gas-particle flow in a two-dimensional channel with a rough wedge step. Taking into account that scattering of particles, reflected from a rough surface, depends on the particle shape very weakly, we consider only spherical particles.

Let us assume that the particle size distribution in the main stream is described by the log-normal law (this law is often used to represent the size of solid particles). In this case the particle mass frequency distribution function has the form

$$
g_{\infty}\left(r_{\mathrm{p}}\right)=\frac{1}{\sqrt{2 \pi} r_{\mathrm{p}} \ln \sigma} \exp \left[-\left(\frac{\ln r_{\mathrm{p}}-\ln r_{\mu}}{\sqrt{2 \pi} \ln \sigma}\right)^{2}\right] .
$$

Figure 6 shows the plots of this function used in calculations. The most probable particle radius $r_{\mathrm{p} m}$ is the same for both distributions. The flow of monosized particles with radius $r_{\mathrm{p}}=r_{\mathrm{p} m}$ was computed for comparison. The parameter $\sigma$ in Eq. (1) characterizes the deviation of particle sizes. The values $\sigma=1.2$ and $\sigma=1.728$ correspond to the standard deviation 0.182 and 0.546 of the logarithm of the particle radius. The parameter $r_{\mu}$ in Eq. (1) is related with $r_{\mathrm{p} m}$ and $\sigma$ : $r_{\mu}=r_{\mathrm{p} m} / \exp \left(2 \ln ^{2} \sigma\right)$.

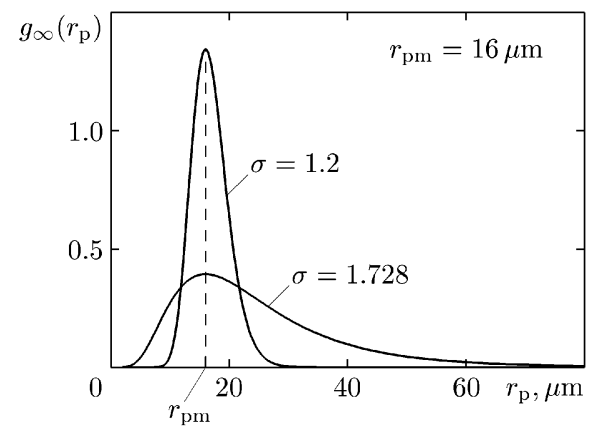

Fig. 6. Particle mass distribution function in the main stream. 


\subsection{Numerical model of the particle-phase flow}

The scheme of the flow is shown in Fig. 7. We assume a low concentration of particles so that we can neglect the effect of collisions between particles and the reverse effect of the particles on the carrier gas flow. By applying to our problem the procedure, described in $\left[{ }^{10}\right]$ for a uniform flow over a body, we can estimate the upper limit of the particle concentration in the main stream when these assumptions are valid. In terms of the particle volume fraction $\alpha_{\mathrm{p} \infty}$, these estimates for coarse-grained monosized particles can be expressed as follows: the inter-particle collisions are negligible when $\alpha_{\mathrm{p} \infty} \lesssim r_{\mathrm{p}} \cos \beta /(30 \Delta)$ and the twoway coupling effects are negligible when $\alpha_{\mathrm{p} \infty} \lesssim r_{\mathrm{p}} /(4 \Delta)(\beta$ and $\Delta$ are shown in Fig. 7). For the used values $r_{\mathrm{p}}=16 \mu \mathrm{m}, \beta=15^{\circ}$ and $\Delta=0.2 \mathrm{~m}$, the first estimation gives $\alpha_{\mathrm{p} \infty} \lesssim 3 \times 10^{-6}$, and the second one $\alpha_{\mathrm{p} \infty} \lesssim 2 \times 10^{-5}$. Thus the model of dilute one-way coupled flow is valid in our problem if $\alpha_{\mathrm{p} \infty} \lesssim 3 \times 10^{-6}$.

At first, we solved numerically the Euler equations to determine the carrier gas flow field (a TVD-scheme of the second order was used), and then calculated the particle trajectories.

The system of equations, describing the motion of an individual particle includes the momentum and angular momentum equations and the kinematic relation for the particle radius-vector $\boldsymbol{r}$ :

$$
m_{\mathrm{p}} \frac{\mathrm{d} \boldsymbol{V}_{\mathrm{p}}}{\mathrm{d} t}=\boldsymbol{f}_{\mathrm{D}}+\boldsymbol{f}_{\mathrm{M}}, \quad J_{\mathrm{p}} \frac{\mathrm{d} \boldsymbol{\omega}_{\mathrm{p}}}{\mathrm{d} t}=\boldsymbol{l}_{\mathrm{p}}, \quad \frac{\mathrm{d} \boldsymbol{r}}{\mathrm{d} t}=\boldsymbol{V}_{\mathrm{p}} .
$$

Here $m_{\mathrm{p}}, J_{\mathrm{p}}, \boldsymbol{V}_{\mathrm{p}}$, and $\boldsymbol{\omega}_{\mathrm{p}}$ are the mass, the moment of inertia, the translational velocity and the angular velocity of a particle, respectively. The aerodynamic drag force $f_{\mathrm{D}}$, the Magnus force $f_{\mathrm{M}}$, and the damping torque $l_{\mathrm{p}}$, acting on a particle from the carrier gas, are defined as

$$
\begin{aligned}
\boldsymbol{f}_{\mathrm{D}} & =\frac{1}{2} C_{\mathrm{D}} \pi r_{\mathrm{p}}^{2} \rho\left|\boldsymbol{V}-\boldsymbol{V}_{\mathrm{p}}\right|\left(\boldsymbol{V}-\boldsymbol{V}_{\mathrm{p}}\right), \\
\boldsymbol{f}_{\mathrm{M}} & =\frac{4}{3} C_{\boldsymbol{\omega}} \pi r_{\mathrm{p}}^{3} \rho\left[\left(\boldsymbol{\omega}-\boldsymbol{\omega}_{\mathrm{p}}\right) \times\left(\boldsymbol{V}-\boldsymbol{V}_{\mathrm{p}}\right)\right], \\
\boldsymbol{l}_{\mathrm{p}} & =\frac{1}{2} C_{l} r_{\mathrm{p}}^{5} \rho\left|\boldsymbol{\omega}-\boldsymbol{\omega}_{\mathrm{p}}\right|\left(\boldsymbol{\omega}-\boldsymbol{\omega}_{\mathrm{p}}\right) .
\end{aligned}
$$

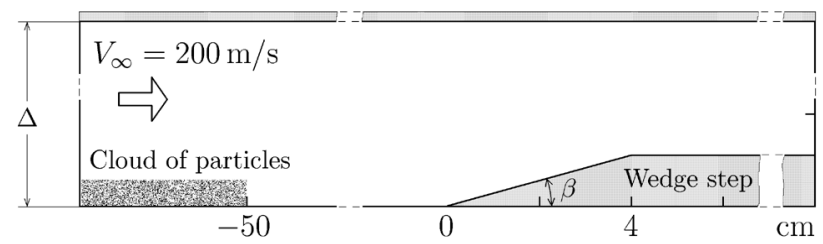

Fig. 7. Scheme of flow in a channel. 
The drag coefficient $C_{\mathrm{D}}$ was calculated from the Henderson approximate correlations [ $\left.{ }^{11}\right]$. For the case of a subsonic flow regime over a particle $\left(M_{\mathrm{p}}<1\right)$ and negligibly small difference between temperatures of the particle and the carrier gas $\left(T_{\mathrm{p}} / T=1\right.$, such conditions were realized in the considered two-phase flow), the formula for $C_{\mathrm{D}}$ takes the form

$$
\begin{gathered}
C_{\mathrm{D}}=24\left[R e_{\mathrm{p}}+M_{\mathrm{p}} \sqrt{\frac{\gamma}{2}}\left\{4.33+\frac{2.12}{1.353} \times \exp \left(-0.247 \frac{R e_{\mathrm{p}}}{M_{\mathrm{p}}} \sqrt{\frac{2}{\gamma}}\right)\right\}\right]^{-1} \\
+\exp \left(-\frac{M_{\mathrm{p}}}{2 \sqrt{R e_{\mathrm{p}}}}\right)\left[\frac{4.5+0.38\left(0.03 R e_{\mathrm{p}}+0.48 \sqrt{R e_{\mathrm{p}}}\right)}{1+0.03 R e_{\mathrm{p}}+0.48 \sqrt{R e_{\mathrm{p}}}}\right. \\
\left.+0.1 M_{\mathrm{p}}^{2}+0.2 M_{\mathrm{p}}^{8}\right]+0.6 M_{\mathrm{p}} \sqrt{\frac{\gamma}{2}}\left[1-\exp \left(-\frac{M_{\mathrm{p}}}{R e_{\mathrm{p}}}\right)\right] \\
\operatorname{Re}_{\mathrm{p}}=\frac{2 r_{\mathrm{p}}\left|\boldsymbol{V}-\boldsymbol{V}_{\mathrm{p}}\right|}{\nu}, \quad M_{\mathrm{p}}=\frac{\left|\boldsymbol{V}-\boldsymbol{V}_{\mathrm{p}}\right|}{a}
\end{gathered}
$$

where $\nu$ is the kinematic viscosity and $a$ the sound velocity. The Magnus force coefficient $C_{\omega}$ depends on the parameter $\gamma_{\omega}=r_{\mathrm{p}}\left|\boldsymbol{\omega}-\boldsymbol{\omega}_{\mathrm{p}}\right| /\left|\boldsymbol{V}-\boldsymbol{V}_{\mathrm{p}}\right|$ and it was calculated using the relation suggested in $\left[{ }^{12}\right]$ for the case of $2 \gamma_{\omega} \geq 0.45$ and was taken equal to $3 / 4$ otherwise:

$$
C_{\omega}= \begin{cases}\frac{3}{8 \gamma_{\omega}}\left[0.45+\left(2 \gamma_{\omega}-0.45\right) \exp \left(-0.075 \gamma_{\omega}^{0.4} R e_{\mathrm{p}}^{0.7}\right)\right], & 2 \gamma_{\omega} \geq 0.45 \\ \frac{3}{4}, & 2 \gamma_{\omega}<0.45\end{cases}
$$

The damping torque coefficient $C_{l}$ was determined using the approximate formula from $\left[{ }^{13}\right]$ :

$$
C_{l}=\frac{C_{l 1}}{\sqrt{R e_{\mathrm{p} \omega}}}+\frac{C_{l 2}}{R e_{\mathrm{p} \omega}}=\frac{1}{R e_{\mathrm{p} \omega}}\left(C_{l 1} \sqrt{R e_{\mathrm{p} \omega}}+C_{l 2}\right), \quad R e_{\mathrm{p} \omega}=\frac{r_{\mathrm{p}}^{2}\left|\boldsymbol{\omega}-\omega_{\mathrm{p}}\right|}{\nu},
$$

where the values of $C_{l 1}$ and $C_{l 2}$ for different ranges of $R e_{\mathrm{p} \omega}$ are as follows:

$$
\begin{array}{ccccc}
R e_{\mathrm{p} \omega} & 0-6 & 6-20 & 20-50 & 50-4 \times 10^{4} \\
C_{l 1} & 0 & 5.32 & 6.44 & 6.45 \\
C_{l 2} & 16 \pi & 37.2 & 32.2 & 32.1
\end{array}
$$


Particles in the undisturbed main stream were assumed to be distributed uniformly, their translational velocity was taken equal to the gas velocity $V_{\infty}$ and the angular velocity was zero.

A modified Crowe method (original version is described in $\left[{ }^{14}\right]$ ) was used to compute the distribution of the particle concentration in the flow. We now briefly explain the method. Let us consider in an undisturbed flow a particle cloud with a height of $\Delta$ and a length of $L_{\infty}$, containing $N_{\mathrm{p}}$ particles. Divide the calculation domain into cells by introducing a regular grid. The value of $L_{\infty}$ together with the main stream velocity $V_{\infty}$ determines the scale of time averaging $T=L_{\infty} / V_{\infty}$. Let $\tau_{k i j}$ be the residence time of the $k$ th particle in the cell $i, j\left(\tau_{k i j}=0\right.$, if the trajectory of this particle does not go through this cell). The time-averaged particle density $n_{\mathrm{p} i j}$ in the cell $i, j$ of volume $\Omega_{i j}$ can be calculated as follows:

$$
n_{\mathrm{p} j}=\frac{1}{\Omega_{i j} T} \sum_{k=1}^{N_{\mathrm{p}}} \tau_{k i j} .
$$

In a similar way the particle volume fraction in this cell is determined as

$$
\alpha_{\mathrm{p} i j}=\frac{1}{\Omega_{i j} T} \sum_{k=1}^{N_{\mathrm{p}}} \tau_{k i j} r_{\mathrm{p} k}^{3},
$$

where $r_{\mathrm{p} k}$ is the radius of the $k$ th particle ( $r_{\mathrm{p} k}=r_{\mathrm{p}}$ for monosized particles).

Although the particle volume fraction in the initial cloud is taken constant and equal to $\alpha_{\mathrm{p} \infty}$, the locations of particles in this cloud are taken at random. However, random sampling of the particle locations result in some fluctuations of the particle volume fraction in the initial cloud. To decrease the effect of these fluctuations on the resulting field of $\alpha_{\mathrm{p}}$, we use the following correction scheme. The initial cloud is divided into a set of small clouds. Consider the $k$ th particle in the initial cloud and denote $\varepsilon_{k}$ the small cloud inside which the $k$ th particle is located. Let $\alpha_{\mathrm{p} \varepsilon_{k}}$ be the average volume fraction of particles in the cloud $\varepsilon_{k}$. Let

$$
\eta_{\alpha}\left(\varepsilon_{k}\right)=\frac{\alpha_{\mathrm{p} \infty}}{\alpha_{\mathrm{p} \varepsilon_{k}}}
$$

Now the corrected expression for $\alpha_{\mathrm{p}}$ in the cell $i, j$ takes, instead of (8), the form (subscripts $i$ and $j$ are omitted)

$$
\alpha_{\mathrm{p}}=\frac{1}{\Omega T} \sum_{k=1}^{N_{\mathrm{p}}}\left[\tau_{k} r_{\mathrm{p} k}^{3} \cdot \eta_{\alpha}\left(\varepsilon_{k}\right)\right] .
$$

Here the factors $\eta_{\alpha}\left(\varepsilon_{k}\right)$ play the role of weight coefficients. They take into account the difference between $\alpha_{\mathrm{p} \varepsilon_{k}}$ and $\alpha_{\mathrm{p} \infty}$ for small clouds from which the particles travel through the grid cell. 
For the polydisperse particles, the resulting value of $\alpha_{\mathrm{p}}$ in the cell $i, j$ can be found as the sum of the particle concentrations calculated for particles of different size fractions

$$
\alpha_{\mathrm{p}}=\sum_{r=1}^{N_{r}} \alpha_{\mathrm{p} r}
$$

where $N_{r}$ is the total number of particle size fractions used in calculations (subscript $r$ denotes the size fraction), and the volume fraction $\alpha_{\mathrm{p} r}$ of particles of the $r$ th size fraction must be calculated from Eq. (10). In our calculations we used values of $N_{r}$ from 50 to 90 .

\subsection{Discussion of computational results}

The computation domain had dimensions of $1.5 \times 0.2 \mathrm{~m}$, and the inlet boundary was $0.5 \mathrm{~m}$ away from a wedge edge. The carrier gas was air with the following parameters at the inlet cross-section: $V_{\infty}=200 \mathrm{~m} / \mathrm{s}, T_{\infty}=268 \mathrm{~K}, P_{\infty}=10^{5} \mathrm{~Pa}$. The wedge material was a weak steel, and the particle material was corundum (density $3950 \mathrm{~kg} / \mathrm{m}^{3}$ ). The roughness profile of the wedge surface was generated numerically as described in Subsection 2.1. For comparison, we also performed calculations for a wedge with a smooth surface. The top wall of the channel was smooth. Monosized and polydisperse particles with a log-normal size distribution were considered.

Uniform gas-particle flow over all inlet cross-section was assumed when simulating the distribution of the particle volume fraction in the channel. However, for the visualization of the particle-phase flow we followed the motion of particles from the thin (a half of the step height) layer adjacent to the bottom wall of the channel. The flow patterns and the outlet distributions of the relative particle volume fraction are shown in Fig. 8. It is seen that the particle dispersion due to particle size distribution is as important as that due to the scattering of particles, reflected from the rough wedge. The role of size distribution increases with the standard deviation of the particle size, and it becomes prevalent when the deviation is large enough. Note that for $\sigma=1.728$, the distributions of $\alpha_{\mathrm{p}} / \alpha_{\mathrm{p} \infty}$ at the outlet cross-section practically coincide for smooth and rough wedge surfaces. Therefore in this case the role of roughness is negligible compared with that of the particle size distribution. 
Smooth wedge surface
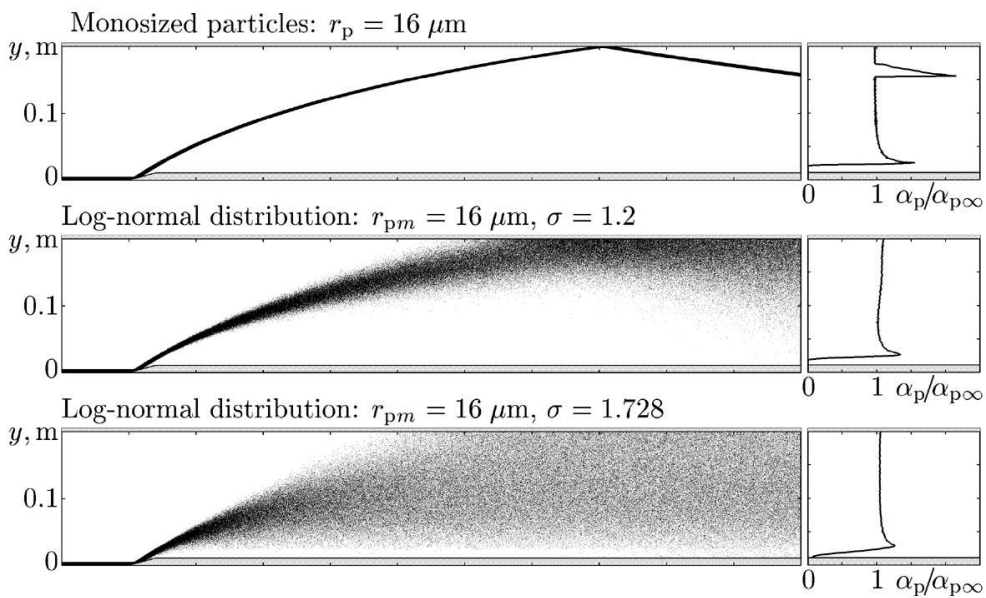

Rough wedge surface
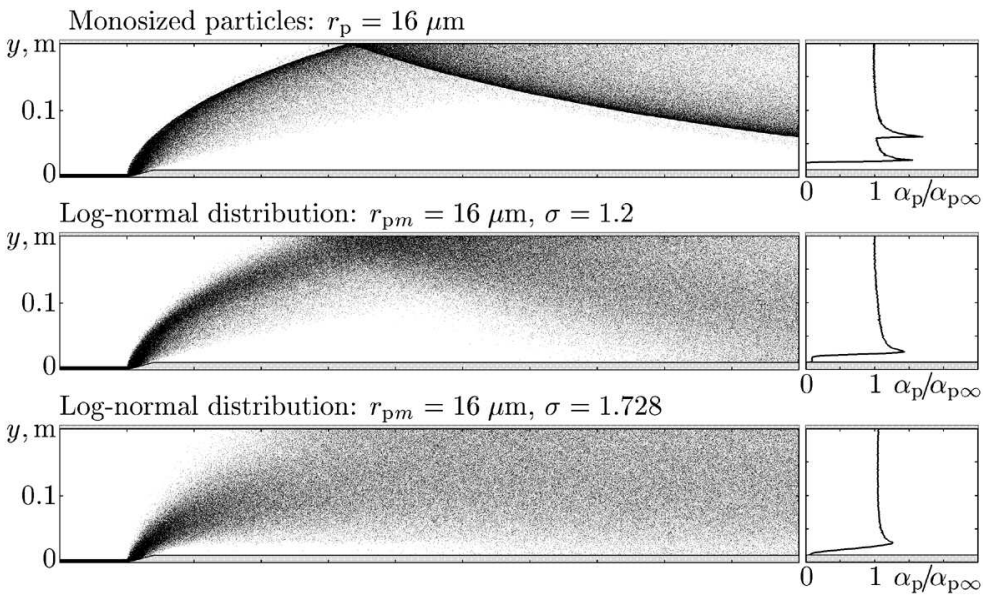

Fig. 8. Instantaneous patterns of particles in the channel flow over a wedge step (left) and profiles of the particle volume fraction at the outlet cross-section of the channel (right) for monosized and log-normal distributed particles.

\section{CONCLUSIONS}

Our analysis leads to two important conclusions.

First, the effect of the non-sphericity of particles (we considered ellipsoids of revolution with small eccentricity, cubic and prismatic particles with the ratio of sides equal to 0.8 ) is negligible compared with that of the erosive roughness of the surface on the scattering of reflected particles. 
Second, the role of particle size distribution (we took the log-normal law) in dispersion of particles downstream the channel flow can become predominant over the effect of the roughness with increasing standard deviation of particle size distribution.

One more essential effect relating to the wedge roughness should be noted. The additional drag force of the wedge in a gas-particle flow due to the particle impacts was calculated for the cases of smooth and rough wedge sides. In the latter case the force was found to be twice as large. This can be explained by the increase of the effective mean angle of particle impacts with the increase of the roughness.

\section{ACKNOWLEDGEMENTS}

This research was supported by the INTAS (grant No. 00-0309) and partly by the Ministry of Education and Science of Russia (grant No. E02-4.0-138).

\section{REFERENCES}

1. Balanin, B. A. and Trakhov, E. P. The experimental equipment for investigation of twophase flows. In The Flow of Viscous and Unviscous Gas. The Two-phase Fluids (Gasdynamics and Heat Transfer, No. 6). Leningrad University Publishing House, Leningrad, 1981, 32-40 (in Russian).

2. Crowe, C. T., Sommerfeld, M. and Tsuji, Y. Multiphase Flow with Droplets and Particles. CRC Press, Boca Raton, 1998.

3. Sommerfeld, M. Modelling of particle-wall collisions in confined gas-particle flows. Int. J. Multiphase Flow, 1992, 18, 905-926.

4. Sommerfeld, M. and Zivkovic, G. Recent advances in the numerical simulation of pneumatic conveying through pipe systems. In Computational Methods in Applied Sciences (Hirsch, Ch., Periaux, J. and Osnate, E., eds.). Elsevier Science, 1992, 201212.

5. Sommerfeld, M., Huber, N. and Wächter, P. Particle-wall collisions: Experimental studies and numerical models. In Gas-Solid Flows (Stock, D. E., Reeks, M. W., Tsuji, Y., Gautam, M., Michaelidies, E. E. and Jurewicz, J. T., eds.). ASME FED, 1993, vol. 166, 183-191.

6. Schade, K.-P. and Hädrich, Th. Investigation of influence of wall roughness on particlewall collision. In Proc. Third International Conference on Multiphase Flow. Lyon, 1998 (CD-ROM ICMF'98, paper 250).

7. Tsirkunov, Yu. M. and Panfilov, S. V. Modelling of particle-wall interaction in two-phase flows at moderate and high particle impact velocity. In Proc. Third International Conference on Multiphase Flow. Lyon, 1998 (CD-ROM ICMF'98, paper 693).

8. Tsirkunov, Yu. M., Panfilov, S. V. and Klychnikov, M. B. Semiempirical model of impact interaction of a disperse impurity particle with a surface in a gas suspension flow. $J$. Eng. Phys. Thermophys., 1994, 67, 1018-1025.

9. Sommerfeld, M. Kinetic simulation for analysing the wall collision process of nonspherical particles. In Proc. ASME FEDSM'02. Montreal, Quebec, 2002 (CD-ROM, paper 31239).

10. Tsirkunov, Yu. M. Gas-particle flows around bodies - key problems, modeling and numerical analysis. In Proc. Fourth International Conference on Multiphase Flow. New Orleans, 2001 (CD-ROM ICMF'2001, paper 609). 
11. Henderson, C. B. Drag coefficients of spheres in continuum and rarefied flows. AIAA J., 1976, 14, 707-708.

12. Oesterlé, B. and Bui Dinh, T. Experiments on the lift of a spinning sphere in a range of intermediate Reynolds numbers. Exp. Fluids, 1998, 25, 16-22.

13. Dennis, S. C. R., Singh, S. N. and Ingham, D. B. The steady flow due to a rotating sphere at low and moderate Reynolds numbers. J. Fluid Mech., 1980, 101, 257-279.

14. Crowe, C. T. Review - numerical models for dilute gas-particle flows. ASME J., Fluid Eng., 1982, 104, 297-303.

\title{
Osakeste hajumine seinapõrgetel ja selle mõju tahkefaasi voolule
}

\author{
Yury Tsirkunov ja Sergei Panfilov
}

Matemaatilise modelleerimise meetodil on uuritud osakeste hajumist põrkumisel jäigalt pinnalt. Pinna kareduse ja osakeste mittesfäärilisuse mõju on analüüsitud nii eraldi kui ka üheskoos. On modelleeritud allahelikiiruselist gaasiosakeste voolust kahemõõtmelises kiilukujulise astmega kanalis. Tahkefaasi vooluväli ja kontsentratsiooniprofiilid on saadud karedapinnalise kiilu jaoks, võttes arvesse osakeste jaotust suuruse järgi. Tulemusi on võrreldud voolamisega siledapinnalise kiiluga kanalis monofraktsiooniliste osakeste korral. 